\title{
Towards Data-Driven Hospital Antimicrobial Stewardship: Secondary use of Routine Electronic Care and Prescribing Records to Inform Antimicrobial Stewardship Programmes
}

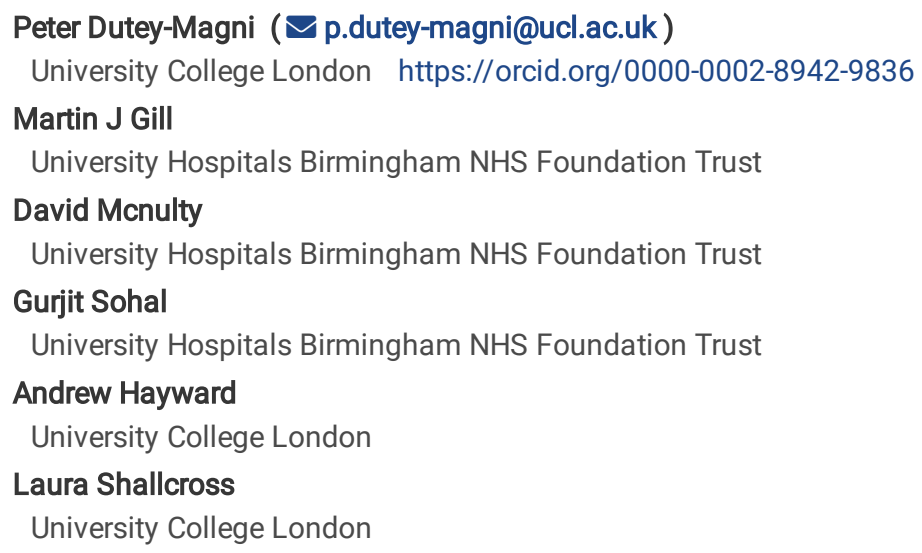

Research

Keywords: antimicrobial stewardship, medical informatics applications, electronic health records, electronic prescribing, public health surveillance, epidemiological monitoring, health information systems, bacterial infections and mycoses

Posted Date: June 4th, 2020

DOl: https://doi.org/10.21203/rs.3.rs-33072/v1

License: @ (i) This work is licensed under a Creative Commons Attribution 4.0 International License. Read Full License 


\section{Abstract \\ Background}

Hospital antimicrobial stewardship (AMS) programmes are multidisciplinary initiatives to optimise the use of antimicrobials. Most AMS programmes rely on time-consuming manual audits to monitor clinicians' antibiotic prescribing practice. Much of the information needed is already contained within electronic health records (EHRs) and could be used to inform AMS programmes. The objective of this study was to evaluate the feasibility of building analytics from hospital EHRs to facilitate AMS interventions.

\section{Methods}

This study used electronic prescribing, laboratory and clinical coding records of adult patients admitted to six specialties at Queen Elizabeth Hospital, Birmingham, UK between September 2017 and August 2018 ( $n=61,679$ admissions). Duration of prescribing, compliance with clinical guidelines, and timing of switch from intravenous to oral antibiotics in relation to clinical progression were assessed. Outputs were visualised at ward, specialty and consultant levels.

\section{Results}

A total of 59,884 antibacterial prescription records were linked into 24,653 therapy episodes. Among the $35 \%$ of admissions prescribed an antibacterial, the mean days of therapy per admission was 4.3 days and 9.9 days in elective and emergency hospitalisations respectively. Twentytwo percent (207/997) of therapy episodes for low-severity community-acquired pneumonia were initiated with the antibiotic recommended by locally-approved prescribing guidelines. Data suggested average delays in switching from intravenous to oral therapy of up to 3.6 days [95\% Cl: 3.4 ; 3.7]. Microbial cultures were performed prior to treatment initiation in just $22 \%$ of antibacterial prescriptions.

\section{Conclusions}

Hospital EHRs can be used to construct meaningful measures of antibiotic stewardship, demonstrating potential value for audit and feedback interventions.

\section{Introduction}

Antimicrobial stewardship (AMS) aims 'first, to ensure effective treatment of patients with infection, and second, to minimise collateral damage from antimicrobial use' [1]. Clinical guidelines [2-7] advocate regular clinical audits of prescriptions and feedback of results to prescribers by infection specialists. Determining prescribing quality, for example by assessing compliance with guidelines, is both labour-intensive and dependent on specialist expertise [8]. Point prevalence surveys of antibiotic prescribing are prohibitive in terms of professional time and methodological skill. Hospitals thus lack the capacity to undertake these approaches on a sufficient scale [8-10] and at regular intervals.

Electronic health records (EHRs) collected routinely by hospital information systems offer potential solutions to this problem. King et al. [11] and Hand et al. [12] scoped the potential role of electronic prescribing software in supporting prescribers across the full antibiotic lifecycle (prescription initiation; review; discontinuation and dispensing of discharge medications). Other studies have demonstrated the feasibility of using computerised laboratory results, including microbial cultures and sensitivities, to guide the choice of antimicrobial agent in empirical therapy [13] and increase the proportion of cases treated with effective antimicrobials [14].

EHRs thus have the potential to enable a range of functions recommended in AMS guidelines [2], particularly: audit of practice, feedback to prescribers, and infection surveillance. Despite this, the use of EHRs to drive AMS programmes remains modest. Extraction of records remains challenging [15], resulting in very limited secondary use for evidence-based medicine [16].

The aim of the present paper was to assess the feasibility of auditing antimicrobial stewardship practices using routinely-collected EHRs in order to provide relevant information to different AMS stakeholders including clinicians, hospital managers and policy-makers. Key objectives were to:

1. infer the indication of antibiotics prescribed to inpatients

2. assess the congruence of individual prescriptions with local prescribing guidelines, particularly in relation to empirical therapy

3. compute metrics of stewardship beyond consumption of antibiotics

4. compare these metrics between specialties and between consultant teams within specialities.

This feasibility study followed the three steps. First, we synthesised relevant antimicrobial stewardship terminology and concepts from clinical guidelines and infection surveillance protocols, and translated them into operational definitions applicable to EHRs. Second, we modelled and 
visualised records to refine definitions that could be applied to data from one specialist hospital in Birmingham, UK. Third, we analysed EHRs and reviewed compliance of clinical practices with AMS guidelines.

\section{Materials And Methods}

\subsection{Concepts and definitions}

We identified relevant definitions and standards of care from international hospital antimicrobial stewardship and infection treatment guidelines from a list systematically compiled in 2018 [17], alongside four UK-specific reference sources [2, 18-20]. LS, PD and MJG narrowed down a list of concepts on the basis of (a) their relevance to inform a hospital AMS strategy, and (b) the availability of sufficient information to measure them within EHRs. These concepts are further characterised in detail and mapped onto relevant SNOMED-CT concept codes [21] in Additional file 1.

\subsection{Electronic care records}

Queen Elizabeth Hospital Birmingham (QEHB) is a specialist teaching hospital in Birmingham, UK with over 1,000 general and acute inpatient beds. DMcN extracted and pseudonymised EHRs for adult inpatients admitted at QEHB between 2008 and 2019: patient demographic information, clinical diagnosis codes (ICD-10 [22]), clinical procedure codes (OPCS-4 [23]), episodes of care (pseudonymised consultant code, consultant specialty) and ward movements, key investigation results (blood counts, vital signs, blood pressure, renal function, etc.). Antibacterial drug prescription and administration records were extracted from QEHB's locally-developed Prescribing, Information and Communication System (PICS) [24]. PICS is designed as per the common UK 'dose-based' prescribing approach [25], in which prescribers issue a request containing one or more drug names (Trade Family), dose, route and frequency.

MJG extracted all microbial culture results, including no growth results and cultures ordered by general practitioners, applied EUCAST interpretative criteria [26], and classified bacterial isolates by multi-drug resistance profile (multiple, extensive, and pan-drug resistance) according to rules set out by Magiorakos et al. [27].

Quantitative results reported in this paper are restricted to a subset of the entire data, corresponding to episodes of care in six specialties (general medicine, respiratory medicine, geriatric medicine, cardiology, general surgery, urology) for adult inpatients admitted between 1 September 2017 to 31 August 2018 ( $n=61,679$ admissions).

\subsection{Data modelling and visualisation}

PDM wrote data management programmes in Structured Query Language (SQL), R and tidyverse [28-30] and mapped drug names to SNOMED CT UK Drug Extension concepts [21]. A web app developed using R Shiny and JavaScript libraries [31-35] enabled data visualisation on an interactive 'antimicrobial timeline' gathering prescriptions, microbial cultures, sepsis red flags, and clinical diagnoses in a single view (Fig. 1). The timeline is accompanied by a range of biomarker charts (Additional file 2). These include relevant clinical parameters such as white blood cell counts, Creactive protein concentrations. CURB-65 [36], an important risk stratification score for community-acquired pneumonia, was computed without the confusion score due to lack of reliable data.

Note

OOF: one-off, BD: twice per day; TDS: three times per day.

1. Screenshot of antimicrobial timeline visualisation

\subsection{Construction of prescribing episodes}

Graph theory principles were used to construct periods of uninterrupted antibiotic therapy (therapy episodes) by linking related prescription records. Rule definitions underpinning this linkage are available along with this paper (Additional file 3 ). This enabled identification of sequences of drug administration making up therapy episodes, particularly transition from one class of antimicrobials to another. Defined daily doses, days of therapy, and length of therapy (duration of the episode, irrespective of the number of antimicrobials administered concurrently) were calculated and aggregated by ward, specialty, consultant teams, and clinical indication as per definitions by Ibrahim et al. [37].

For each antimicrobial therapy episode, a dynamic table could be constructed with an hourly resolution capturing changes in therapy in relation to clinical parameters (Table 1) as the basis for adjudicating compliance with stewardship metrics.

1. Example structure of a therapy table 


\begin{tabular}{|c|c|c|c|c|c|c|c|c|}
\hline patient & time & mode & last WBC & WBC trend $72 \mathrm{~h}$ & peak CRP in last 72 hours & Last CRP & $\ldots$ & ABCD criteria met? \\
\hline$x$ & 2018-07-31 18:49:51 & IV & 11.0 & -0.05 & 151 & 100 & $\ldots$ & Yes \\
\hline$x$ & 2018-07-31 19:49:51 & IV & 8.2 & -0.02 & 151 & 100 & $\cdots$ & Yes \\
\hline $\mathrm{X}$ & 2018-07-31 20:49:51 & oral & 8.2 & -0.02 & 151 & 40 & $\ldots$ & Yes \\
\hline$\ldots$ & $\ldots$ & $\ldots$ & $\ldots$ & $\ldots$ & $\ldots$ & $\ldots$ & $\ldots$ & $\ldots$ \\
\hline
\end{tabular}

For instance, we computed the time by which criteria for switching from intravenous to oral regimes were met, based on a set of 'ABCD' criteria listed in QEHB's antimicrobial prescribing guidelines (Table 2). Out of these, ability to take oral medication (criterion B) could not be assessed from records, but other criteria could be measured continuously.

1. ABCD criteria: Considerations for intravenous to oral switch (see detailed criteria in Additional files 4 and 5)

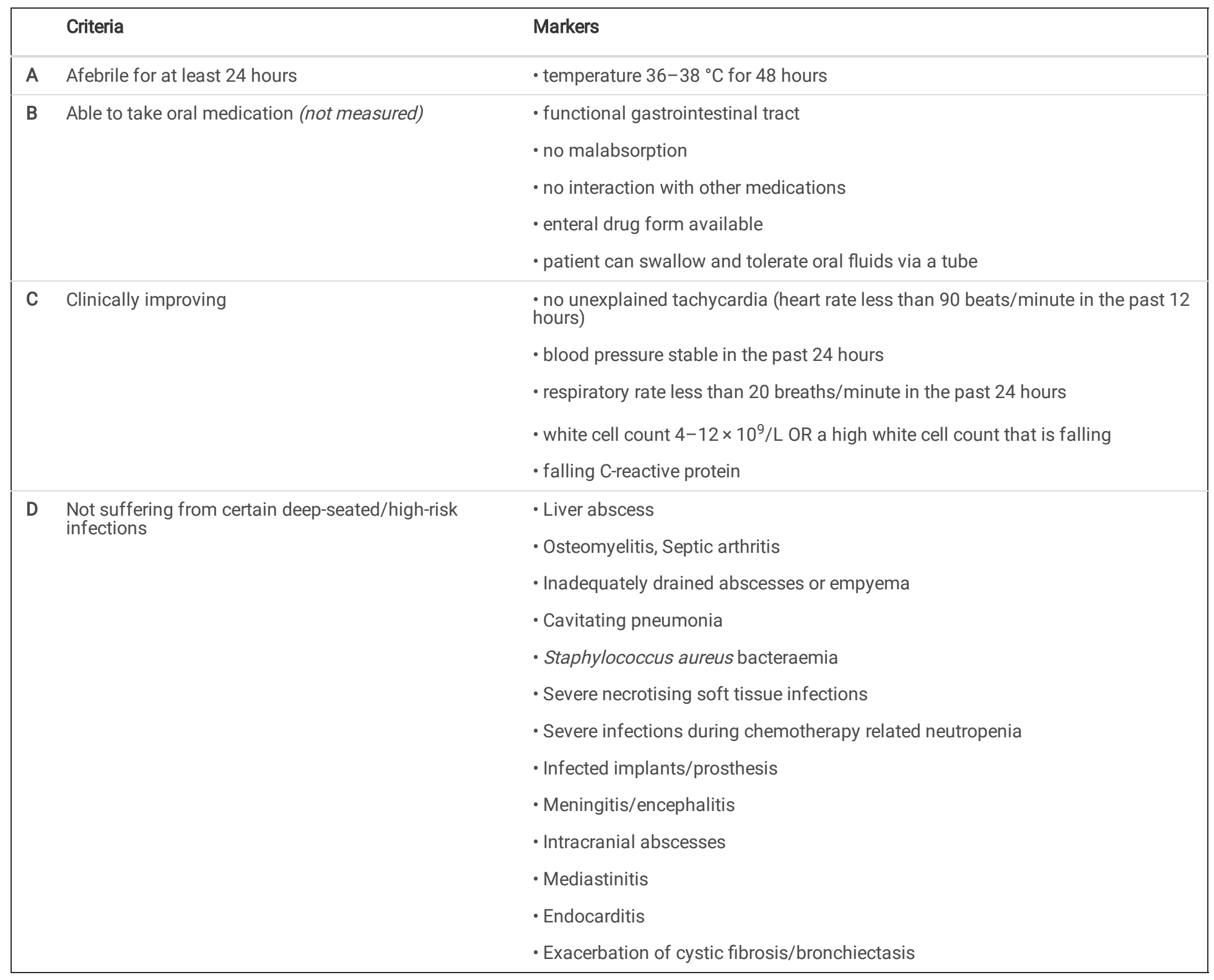

\subsection{Clinical indication training dataset}

PICS captures drug prescription indications as free text. Such information was not made available to researchers due the data containing identifiable information and the high prevalence of missing data (in the region of $50 \%$ ). In order to demonstrate our approach, drug indications were 
instead classified retrospectively using training data. Between 2012 and 2017, a team of pharmacists led and trained by SG performed a standardised, electronically-recorded audit of antibacterials in the following specialties: general medicine, respiratory medicine, geriatric medicine, and general surgery. They reviewed information from PICS and paper clinical notes for a total of 4,200 prescriptions issued for 2,712 patients, classifying each of them into 21 possible indications including 'Not specified' and 'Other'. Of those records, 463 did not have a valid clinical indication, and 364 could not be linked to electronic prescription records, restricting the analysis to a total of 3,228 prescriptions corresponding to 2,901 therapy episodes. Indication categories with fewer than 50 episodes (endocarditis, bronchiectasis, diabetic foot and/or osteomyelitis, surgical prophylaxis) were reclassified as 'Other'. These records were used as training data to predict the clinical indication across all antimicrobial prescriptions, using random forest classification with a moderate-to-low balanced accuracy of $59 \%$ overall. Predictive analytics were estimated using repeated 5 -fold stratified cross-validation and are reported in Additional file 6.

\section{Results}

\subsection{Antimicrobial consumption descriptive characteristics}

Between 1 September 2017 and 31 August 2018, there were 61,679 adult admissions (46,853 distinct patients) across the six specialties. Table 3 presents further admission and prescription characteristics by age group. Across all admissions, the mean length of stay was 4.2 days, and 21,757 (35\%) contained at least one antibacterial prescription. A total of 59,884 antibacterial prescriptions were issued as part of 24,511 antibacterial therapy episodes, 141 of which spanned more than one admission. The mean length of antibacterial therapy episodes (LOT) was 5.8 days, equivalent to a mean 8.7 days of therapy (DOT) per admission. The mean days of therapy was significantly higher (9.9 days) in emergency admissions than in elective admissions (4.3 days, Fig. 2).

Therapy episodes could be analysed to study changes in antibacterial treatment choices and their timing relative to microbiological outcomes and clinical progression. One such change, de-escalation, is recommended when microbial culture and susceptibilities are available, or when there is limited evidence of infection. It is most easily measured in antibiotics with the broadest spectrum of activity, where only a small number of other drugs would have equivalent spectrum.

For instance, therapy episodes initiated with meropenem $(n=969)$ were most commonly:

1. (a) stopped (33\%), after a mean duration of 3.0 days;

2. (b) continued (28\%), after a mean duration of 2.0 days;

3. (c) switched to piperacillin + tazobactam (12\%), after a mean duration of 1.1 days;

4. (d) switched to co-amoxiclav (9.1\%), after a mean duration of 1.9 days;

Outcomes (c) and (d) can be regarded as de-escalation in this particular instance. It is comparatively more difficult to envisage general rules for identifying de-escalation in more common antibiotics, due to variation in local prescribing guidelines and practices.

1. Characteristics of admissions and antibacterial therapy by age group in six selected specialities (September 2017-August 2018) 


\begin{tabular}{|c|c|c|c|c|c|c|c|c|c|c|c|c|}
\hline \multirow{4}{*}{$\begin{array}{l}\text { Age } \\
\text { group }\end{array}$} & \multicolumn{4}{|c|}{ ALL ADMISSIONS } & \multicolumn{8}{|c|}{ ADMISSIONS WITH $\geq 1$ PRESCRIPTION(S) } \\
\hline & Admissions & Mean & LOS & DOT & Admissions & Mean & LOS & Prescriptions & Therapy & Mean & LOT & Mean \\
\hline & $\mathrm{N}$ & $\begin{array}{l}\text { LOS } \\
\text { (SD) }\end{array}$ & IQR & $\begin{array}{l}1,000 \\
\text { bed- } \\
\text { days }\end{array}$ & $\begin{array}{l}\mathrm{N} \text { (\% total } \\
\text { admissions) }\end{array}$ & $\begin{array}{l}\text { LOS } \\
\text { (SD) }\end{array}$ & IQR & $\mathrm{N}$ & $\mathrm{N}$ & $\begin{array}{l}\text { LOT } \\
\text { (SD) }\end{array}$ & IQR & (SD) \\
\hline & & & & $\begin{array}{l}{[95 \%} \\
\mathrm{Cl}]\end{array}$ & & & & & & & & \\
\hline $\begin{array}{l}18- \\
24 \text { years }\end{array}$ & 3,937 & $\begin{array}{l}1.9 \\
(6.0)\end{array}$ & $\begin{array}{l}0.2- \\
1.7\end{array}$ & $\begin{array}{l}788 \\
{[787-} \\
788]\end{array}$ & $1,020(26)$ & $\begin{array}{l}4.6 \\
(10.6)\end{array}$ & $\begin{array}{l}0.8- \\
4.7\end{array}$ & 2,294 & 1,069 & $\begin{array}{l}4.1 \\
(7.5)\end{array}$ & $\begin{array}{l}1.0- \\
4.4\end{array}$ & $\begin{array}{l}5.7 \\
(15.5)\end{array}$ \\
\hline $\begin{array}{l}25- \\
34 \text { years }\end{array}$ & 5,626 & $\begin{array}{l}2.2 \\
(6.8)\end{array}$ & $\begin{array}{l}0.2- \\
1.8\end{array}$ & $\begin{array}{l}789 \\
{[789-} \\
790]\end{array}$ & $1,439(26)$ & $\begin{array}{l}5.8 \\
(12.0)\end{array}$ & $\begin{array}{l}0.9- \\
5.9\end{array}$ & 3,523 & 1,541 & $\begin{array}{l}5.1 \\
(10.5)\end{array}$ & $\begin{array}{l}1.0- \\
5.2\end{array}$ & $\begin{array}{l}6.9 \\
(16.2)\end{array}$ \\
\hline $\begin{array}{l}35- \\
44 \text { years }\end{array}$ & 6,389 & $\begin{array}{l}2.5 \\
(6.9)\end{array}$ & $\begin{array}{l}0.2- \\
2.0\end{array}$ & $\begin{array}{l}795 \\
{[794-} \\
795]\end{array}$ & $1,617(25)$ & $\begin{array}{l}6.6 \\
(11.7)\end{array}$ & $\begin{array}{l}1.0- \\
7.4\end{array}$ & 4,176 & 1,748 & $\begin{array}{l}5.7 \\
(9.8)\end{array}$ & $\begin{array}{l}1.0- \\
6.4\end{array}$ & $\begin{array}{l}7.8 \\
(15.2)\end{array}$ \\
\hline $\begin{array}{l}45- \\
54 \text { years }\end{array}$ & 8,423 & $\begin{array}{l}2.7 \\
(7.2)\end{array}$ & $\begin{array}{l}0.2- \\
2.3\end{array}$ & $\begin{array}{l}827 \\
{[827-} \\
827]\end{array}$ & $2,307(27)$ & $\begin{array}{l}7.1 \\
(12.0)\end{array}$ & $\begin{array}{l}1.2- \\
8.2\end{array}$ & 5,965 & 2,523 & $\begin{array}{l}5.8 \\
(11.7)\end{array}$ & $\begin{array}{l}1.0- \\
6.3\end{array}$ & $\begin{array}{l}8.3 \\
(21.1)\end{array}$ \\
\hline $\begin{array}{l}55- \\
64 \text { years }\end{array}$ & 9,977 & $\begin{array}{l}3.6 \\
(8.4)\end{array}$ & $\begin{array}{l}0.2- \\
3.5\end{array}$ & $\begin{array}{l}834 \\
{[833-} \\
834]\end{array}$ & $3,281(33)$ & $\begin{array}{l}8.5 \\
(12.5)\end{array}$ & $\begin{array}{l}1.6- \\
9.8\end{array}$ & 8,989 & 3,707 & $\begin{array}{l}6.2 \\
(10.9)\end{array}$ & $\begin{array}{l}1.1- \\
7.0\end{array}$ & $\begin{array}{l}9.2 \\
(18.0)\end{array}$ \\
\hline $\begin{array}{l}65- \\
74 \text { years }\end{array}$ & 11,230 & $\begin{array}{l}4.4 \\
(9.5)\end{array}$ & $\begin{array}{l}0.2- \\
4.7\end{array}$ & $\begin{array}{l}740 \\
{[740-} \\
740]\end{array}$ & $4,277(38)$ & $\begin{array}{l}8.9 \\
(13.2)\end{array}$ & $\begin{array}{l}1.8- \\
10.3\end{array}$ & 11,620 & 4,868 & $\begin{array}{l}5.6 \\
(7.8)\end{array}$ & $\begin{array}{l}1.3- \\
7.0\end{array}$ & $\begin{array}{l}8.5 \\
(15.1)\end{array}$ \\
\hline $\begin{array}{l}75- \\
84 \text { years }\end{array}$ & 9,815 & $\begin{array}{l}6.3 \\
(11.6)\end{array}$ & $\begin{array}{l}0.4- \\
7.4\end{array}$ & $\begin{array}{l}674 \\
{[674-} \\
674]\end{array}$ & $4,440(45)$ & $\begin{array}{l}10.7 \\
(14.8)\end{array}$ & $\begin{array}{l}2.1- \\
13.5\end{array}$ & 12,900 & 5,164 & $\begin{array}{l}5.9 \\
(7.1)\end{array}$ & $\begin{array}{l}2.0- \\
7.4\end{array}$ & $\begin{array}{l}9.4 \\
(12.9)\end{array}$ \\
\hline $\begin{array}{l}85- \\
94 \text { years }\end{array}$ & 5,668 & $\begin{array}{l}8.6 \\
(13.1)\end{array}$ & $\begin{array}{l}0.8- \\
11.0\end{array}$ & $\begin{array}{l}618 \\
{[617-} \\
618]\end{array}$ & $2,986(53)$ & $\begin{array}{l}12.9 \\
(15.4)\end{array}$ & $\begin{array}{l}2.8- \\
17.0\end{array}$ & 9,196 & 3,558 & $\begin{array}{l}6.2 \\
(6.1)\end{array}$ & $\begin{array}{l}2.0- \\
8.1\end{array}$ & $\begin{array}{l}10.1 \\
(12.2)\end{array}$ \\
\hline $\begin{array}{l}95+ \\
\text { years }\end{array}$ & 614 & $\begin{array}{l}10.2 \\
(13.5)\end{array}$ & $\begin{array}{l}1.0- \\
14.7\end{array}$ & $\begin{array}{l}607 \\
{[606-} \\
608]\end{array}$ & $390(64)$ & $\begin{array}{l}13.4 \\
(14.9)\end{array}$ & $\begin{array}{l}2.6- \\
19.0\end{array}$ & 1,221 & 475 & $\begin{array}{l}5.8 \\
(5.3)\end{array}$ & $\begin{array}{l}2.0- \\
7.3\end{array}$ & $\begin{array}{l}9.7 \\
(11.2)\end{array}$ \\
\hline All ages & 61,679 & $\begin{array}{l}4.2 \\
(9.5)\end{array}$ & $\begin{array}{l}0.2- \\
4.1\end{array}$ & $\begin{array}{l}726 \\
{[726-} \\
726]\end{array}$ & $21,757(35)$ & $\begin{array}{l}9.1 \\
(13.6)\end{array}$ & $\begin{array}{l}1.6- \\
10.7\end{array}$ & 59,884 & 24,653 & $\begin{array}{l}5.8 \\
(8.8)\end{array}$ & $\begin{array}{l}1.3- \\
7.0\end{array}$ & $\begin{array}{l}8.7 \\
(15.6)\end{array}$ \\
\hline
\end{tabular}

1. Mean and $95 \% \mathrm{Cl}$ of the total DOT per admission in patients receiving antimicrobials at any point during an admission (September $2017-$ August 2018)

\subsection{Congruence with prescribing guidelines}

Congruence of prescribing with local guidelines (first-line choice of therapy) was assessed for common infections. For instance, a total of 4,222 therapy episodes were initiated for community-acquired pneumonia (CAP), 4,109 (97\%) of which could be linked with a CURB-65 severity score in the 48 hours before or after antibiotic initiation (assuming a mental confusion score of 0 due to as this information was not recorded electronically). Table 4 below reports antibiotics initiated as first-line therapy in 2,569 low-severity CAP episodes, that is, episodes with a CURB-65 score of 0 or 1 . At the time of prescribing, QEHB guidelines recommended:

- CURB-65 score 0 or 1: amoxicillin; doxycycline (penicillin allergy)

- CURB-65 score 2: amoxicillin + clarithromycin; benzylpenicillin + clarithromycin; moxifloxacin (penicillin allergy)

- CURB-65 score 3+: co-amoxiclav + clarithromycin; moxifloxacin (penicillin allergy)

1. First-line therapy choice in CAP episodes in patients with a CURB-65 score of 0 or 1 


\begin{tabular}{|c|c|c|}
\hline \multirow[b]{2}{*}{ First-line therapy } & \multicolumn{2}{|c|}{$\begin{array}{l}\text { Therapy episodes } \\
\text { n (\% column total) }\end{array}$} \\
\hline & URB $65=0$ & URB-65 = 1 \\
\hline Amoxicillin & $205(22.1)$ & $249(15.5)$ \\
\hline Amoxicillin, clarithromycin & $56(6.0)$ & $104(6.5)$ \\
\hline Azithromycin & $4(0.4)$ & $6(0.4)$ \\
\hline Benzylpenicillin & $2(0.2)$ & $3(0.2)$ \\
\hline Benzylpenicillin, clarithromycin & $14(1.5)$ & $31(1.9)$ \\
\hline Benzylpenicillin, metronidazole & $1(0.1)$ & $0(0.0)$ \\
\hline Ciprofloxacin & $2(0.2)$ & $9(0.6)$ \\
\hline Clarithromycin & $76(8.2)$ & $75(4.7)$ \\
\hline Clarithromycin, co-amoxiclav & $261(28.2)$ & $541(33.8)$ \\
\hline Co-amoxiclav & $104(11.2)$ & $211(13.2)$ \\
\hline Meropenem & $9(1.0)$ & $24(1.5)$ \\
\hline Piperacillin + tazobactam & $2(0.2)$ & $2(0.1)$ \\
\hline Ceftriaxone & $10(1.1)$ & $2(0.1)$ \\
\hline Clarithromycin, moxifloxacin & $2(0.2)$ & $4(0.2)$ \\
\hline Clarithromycin, piperacillin + tazobactam & $4(0.4)$ & $9(0.6)$ \\
\hline Meropenem, vancomycin & $7(0.8)$ & $10(0.6)$ \\
\hline Other & $168(18.1)$ & $322(20.1)$ \\
\hline Total & $927(100)$ & $1,602(100)$ \\
\hline
\end{tabular}

Out of 927 patients whose CURB-65 score can confidently be assumed to be at the most 1 (factoring in the missing mental confusion score), just 207 (22\%) therapy episodes were initiated with the recommended drug, while 331 (36\%) received therapy recommended for higher CURB-65 scores.

The classifier precision for CAP was 80\% (Additional file 5), indicating that one in five episodes classified as CAP potentially had a different indication. For instance, benzylpenicillin and metronidazole is the first-choice treatment option for aspiration pneumonia. Nevertheless, the low proportion of prescriptions that were congruent with guidelines was confirmed by manual inspection of individual cases.

Next, we examined adherence to microbial sampling guidelines recommending submission of bacterial cultures prior to initiation of empirical treatment [18]. We examined the proportion of prescriptions with a microbial sample taken in the three days leading up to antibacterial therapy initiation. Across a total of 59,696 prescriptions ordered by six selected specialties, $22 \%(n=13,210)$ could be linked to at least one specimen sampled from blood, drains, respiratory tract, intravascular devices, central nervous system, aspirates or other tissue or bone samples. Narrowing the criterion to blood samples only, $18 \%(n=10,906)$ of all prescriptions and $38 \%(1,174 / 3,107)$ of prescriptions for meropenem (mainly used to treat bloodstream infections), could be linked to such a sample. Figure 3 reports findings broken down by specialty and consultant team. Considerable variation can be observed, which could be further examined by exploring the indication for the prescription across each specialty/team.

1. Point and $95 \%$ confidence interval estimates of the proportion of prescriptions initiated with a blood culture sampled in the three days leading up to initiation of prescription and/or therapy by consultant team by specialty by drug type in six selected specialities (September 2017August 2018)

\subsection{Switch to oral therapy}

Conversion from intravenous therapy to oral therapy is another commonly recommended stewardship intervention, which can facilitate discharge and reduce some adverse effects of injections [38]. Within 16,688 out of the 24,510 antibacterial therapy episodes, we identified 17,614 episodes consisting of one or more intravenous prescriptions. Overall, $6,404(36 \%)$ of such the intravenous episodes were converted to oral therapy, with a median and mean duration of intravenous treatment of 2.4 days and 3.5 days respectively. On the contrary, 11,210 intravenous episodes (64\%) 
continued with injections until end of therapy, with a median duration of 1.3 days and a mean duration of 3.5 days. As shown in Fig. 4 , variation in the conversion to oral therapy across clinical teams and specialties was evident and can be attributed, at least in part, to case mix. For instance, a likely explanation for cardiology's lower conversion rate (8\%) is that prolonged intravenous therapy is recommended for deep-seated infections such as endocarditis.

1. Point and $95 \%$ confidence interval estimates of the proportion of intravenous therapy converted to oral therapy ranked by consultant team by specialty (September 2017-August 2018)

We sought to analyse the timeliness of conversion from intravenous to oral therapy. Despite the limited scientific evidence on oral conversion criteria, some simple clinical stability criteria have been promoted and included in clinical guidelines. We chose one such set of criteria in place in QEHB, known as ABCD (Table 2). Out of 6,404 intravenous episodes successfully switched, 2,670 (42\%) met A, C and D criteria before oral conversion occurred. Out of 11,210 sequences never switched, 2,682 (21\%) met A, C and D criteria before end of therapy. Across both sets, the delay between criteria being met and end/conversion of therapy had a median of 2.1 days, a mean of 3.6 days [95\% Cl: $3.4 ; 3.7]$, and a standard deviation of 5.7 days, suggesting considerable variation. Figure 5 presents team- and specialty-level mean delays, suggesting once again some differences between consultant teams within specialties.

1. Point and $95 \%$ confidence interval estimates of the mean time (days) elapsed between ABCD criteria being met and conversion to oral therapy, ranked by consultant team by specialty (September 2017-August 2018)

\section{Discussion}

\subsection{Principal findings}

This single-site clinical audit demonstrates a pragmatic approach to draw on electronic prescription, laboratory and hospital care records to provide meaningful measures of AMS. While this approach cannot be used to provide feedback on the appropriateness of individual-level prescribing decisions, it can identify areas of prescribing behaviour where there is scope for improved stewardship. For example, our findings in relation to compliance with guidelines for low severity CAP highlight potential to optimise empirical prescribing for this common clinical infection syndrome. Similarly, the stewardship metrics we have developed provide a means of monitoring variation in prescribing behaviours across consultant teams and specialties, providing scope to: monitor performance; inform the design of stewardship interventions; evaluate their impact; and engage clinical teams in audit and feedback interventions to optimise their prescribing. Our approach also makes it easier for clinicians to visualise and review patients' prescribing and infection history without requiring lengthy exploration of medical notes.

This feasibility study also reveals the challenges associated with assessing congruence with local prescribing guidelines and the complexity of prescribing decisions. Manual review of individual prescribing records led authors to conclude that there is too much ambiguity in electronic health records to confidently assess the appropriateness of prescribing decisions. Greater clinical research is needed in this field to develop evidencebased standards of care for antimicrobial stewardship (for instance on de-escalation [39]), which could be facilitated by observational studies of routine care records.

\subsection{Study strengths and limitations}

This study is novel in attempting to measure clinical constructs that normally require manual audits or point prevalence surveys [19, 40]. We outlined ways of measuring (and benchmarking) quality of stewardship in clinical practice beyond antimicrobial consumption, which is the main method currently used in antimicrobial surveillance [41]. National surveillance systems for prescribing and resistance in secondary care provide high-quality measures of resistance and prescribing for policy-makers, but they do not address the needs of front-line clinicians who require more detailed metrics to identify opportunities to improve their performance. The approach outlined in this study demonstrates the potential for locallydeveloped analytics to address the local needs and stewardship priorities of clinicians using routinely-collected EHRs.

Existing literature contains few examples of EHR research simultaneously analysing electronic prescribing, laboratory and care records outside of intensive care research. To our knowledge, only large bespoke data engineering platforms have achieved this [42-44]. Unlike the present study, such platforms exploit health messages streaming from hospital information systems in real-time: these contain dynamic information, unlike the retrospective view provided from EHRs commonly curated in hospital warehouses. This noteworthy difference has implications: although dynamic health messages can contain greater detail on clinical decisions, their structure and content is more variable across systems and can involve greater investment in the development of dedicated data models and analytical processing. Consequently, these are currently out of reach of most hospitals. The pragmatic approach described in the present paper, although more rudimentary, is potentially more easily adoptable to a wider range of hospitals, provided that interoperable software can be made available.

This single-site feasibility study exhibits several limitations. Gaps in access to dispensing records prevented analysis of "to take away" medications issued at discharge. Similarly, some diagnostics results (for instance chest radiology reports on lung consolidations, a criterion for the diagnosis and treatment of pneumonia) were not available and prevent detailed analysis of diagnostics stewardship. Finally, prescription records obtained 
from a snapshot source did not include a history of changes made to prescriptions' intended duration. This prevented analysis of how frequently prescriptions were stopped early. All analyses were restricted to structured data, and did not attempt to derive information which may have been recorded in free text in medical notes.

\subsection{Implications}

International guidelines recommend investment in surveillance and analytics to rationalise the use of antimicrobials. Electronic prescription, laboratory and hospital care record systems are rapidly becoming commonplace in high-income nations. The UK's Antimicrobial Resistance National Action Plan [45] aims to complete the introduction of electronic prescribing systems across England by 2025, alongside the adoption of international clinical terminology in computerised laboratory systems [46]. Strong evidence supports the use of feedback to prescribers [2, 47], but feedback needs to be relevant, reliable and timely to influence prescribing behaviour [48]. Further research is needed to statistically adjust those measures for case mix in the same way as consumption measures [49]. User-centred research [50] is also needed to tailor these measures to individual clinical teams, or to enable AMS teams or hospital managers to monitor specific prescribing behaviours across hospitals.

\section{Conclusions}

This single-site clinical audit shows it is feasible to draw on electronic prescription, laboratory and hospital care records to provide meaningful measures of AMS, by:

1. Reconstructing 'therapy episodes', which link all relevant prescription records and enable analyses of the length, changes and discontinuation of antimicrobial therapy.

2. Inferring the clinical intent and indication of prescriptions (for both monotherapy and combination therapy). We have illustrated the use of supervised classification in general medicine specialties with moderate accuracy for the most common infection categories.

3. Computing stewardship performance and quality metrics. Examples include conversion of intravenous therapy to oral therapy when patients show signs of resolution, microbial culture sampling and congruence with guidelines.

One of the most significant obstacles hindering hospitals' stewardship efforts lies the difficulty in extracting and analysing EHRs from a range of diverse systems [16]. Reproducible analytical tools are now available to assist microbiology culture and sensitivity analytics [51]. Software development is underway support other hospitals in adopting the approach tested in the present study.

\section{Abbreviations}

\begin{tabular}{|ll|}
\hline AMS & antimicrobial stewardship \\
\hline CAP & community-acquired pneumonia \\
\hline CRP & C-reactive protein \\
\hline DOT & days of therapy \\
\hline EHR & electronic health record \\
\hline IV & intravenous \\
\hline LOT & length of therapy \\
\hline PICS & Prescribing, Information and Communication System \\
\hline QEHB & Queen Elizabeth Hospital Birmingham \\
\hline UK & United Kingdom \\
\hline WBC & white blood cell count \\
\hline
\end{tabular}

\section{Declarations}

\section{Ethics approval and consent to participate}

This research was approved by University College London's Research Ethics Committee (REC reference 16765/002). Informed consent was not sought for the secondary analysis of pseudonymised electronic health records.

\section{Consent for publication}

Not applicable. 


\section{Availability of data and materials}

The pseudonymised datasets analysed during the current study are not publicly available due to residual risk of patient identifiability.

\section{Competing interests}

None to declare.

\section{Authors' contributions}

DMcN extracted data for the study team. MJG extracted and classified microbial culture and sensitivity records. GS led the collection of the prescription review data. PDM programmed and conducted analyses, and drafted the manuscript. MJG, LS, AH and PDM reviewed visualisations of results and interpreted the findings. All authors have read and approved the submitted manuscript.

\section{Acknowledgements}

This work uses pseudonymised data provided by patients and collected by the NHS as part of their care and support. The authors also acknowledge the contribution of staff of the Pharmacy Department at University Hospital Birmingham NHS Foundation Trust, who collected highquality antimicrobial review outcome data.

This paper was published on behalf of the PASS research group: Niall Anderson, Elise Crayton, Gillian Forbes, Arnoupe Jhass, Emma Richardson, Michelle Richardson, Patrick Rockenschaub, Catherine Smith, Elizabeth Sutton, Rosanna Traina (Investigation); Lou Atkins, Anne Conolly, Spiros Denaxas, Ellen Fragaszy, Rob Horne, Patty Kostkova, Fabiana Lorencatto, Susan Michie, Jennifer Mindell, John Robson, Claire Royston, Carolyn Tarrant, James Thomas, Jonathan West (Conceptualisation, Funding Acquisition and Resources); Haydn Williams (Resources); Nadia Elsay, Chris Fuller (Project Administration).

\section{Funding}

This study was carried out as part of Preserving Antibiotics through Safe Stewardship (PASS), a programme grant funded by the Economic \& Social Research Council (grant reference ES/P008321/1).

AH is a National Institute for Health Research (NIHR) Senior Investigator. LS is funded by a NIHR Clinician Scientist Award (CS-2016-007). The views expressed in this article are those of the authors and not necessarily those of the NIHR, or the Department of Health and Social Care.

\section{References}

1. Davey P, Sneddon J, Nathwani D. Overview of strategies for overcoming the challenge of antimicrobial resistance. Expert Rev Clin Pharmacol. 2010;3:667-86.

2. National Institute of Health and Care Excellence. Antimicrobial stewardship: systems and processes for effective antimicrobial medicine use. NICE guideline NG15, https://www.nice.org.uk/guidance/ng15 (2015, accessed 24 October 2018).

3. SARI Hospital Antimicrobial Stewardship Working Group.

Guidelines for Antimicrobial Stewardship in Hospitals in Ireland

SARI Hospital Antimicrobial Stewardship Working Group. Guidelines for Antimicrobial Stewardship in Hospitals in Ireland. Dublin, Ireland. https://www.hpsc.ie/a-z/microbiologyantimicrobialresistance/infectioncontrolandhai/guidelines/File,4116,en.pdf (2009, accessed 11 November 2019).

4. Haute Autorité de Santé. Antibiotic therapy and prevention of bacterial resistance in healthcare organisations. Clinical practice guideline April 2008. Saint-Denis La Plaine, France, https://www.has-sante.fr/jcms/c_665169/fr/strategie-d-antibiotherapie-et-prevention-des-resistancesbacteriennes-en-etablissement-de-sante (2008, accessed 11 November 2019).

5. Pulcini C, Binda F, Lamkang AS, et al. Developing core elements and checklist items for global hospital antimicrobial stewardship programmes: a consensus approach. Clin Microbiol Infect 2018; 1-6.

6. Dellit TH, Owens RC, McGowan JE, et al. Infectious Diseases Society of America and the Society for Healthcare Epidemiology of America Guidelines for Developing an Institutional Program to Enhance Antimicrobial Stewardship. Clin Infect Dis. 2007;44:159-77.

7. Stichting Werkgroep Antibiotica Beleid. A Teams Practical Guide. Antimicrobial Stewardship in the Netherlands, http://www.ateams.nl/depraktijkgids/download-de-praktijkgids (2018, accessed 11 November 2019). 
8. Chung GWW, Wu JEE, Yeo CLL, et al. Antimicrobial stewardship: A review of prospective audit and feedback systems and an objective evaluation of outcomes. Virulence. 2013;4:151-7.

9. Howard P, Pulcini C, Levy Hara G, et al. An international cross-sectional survey of antimicrobial stewardship programmes in hospitals. J Antimicrob Chemother. 2014;70:1245-55.

10. Van Gastel E, Costers M, Peetermans WEE, et al. Nationwide implementation of antibiotic management teams in Belgian hospitals: a selfreporting survey. J Antimicrob Chemother. 2010;65:576-80.

11. King A, Cresswell KM, Coleman JJ, et al. Investigating the ways in which health information technology can promote antimicrobial stewardship: a conceptual overview. J R Soc Med. 2017;110:320-9.

12. Hand KS, Cumming D, Hopkins S, et al. Electronic prescribing system design priorities for antimicrobial stewardship: a cross-sectional survey of 142 UK infection specialists. J Antimicrob Chemother 2016; dkw524.

13. Cánovas-Segura B, Campos M, Morales A, et al. Development of a clinical decision support system for antibiotic management in a hospital environment. Prog Artif Intell. 2016;5:181-97.

14. Curtis CE, Al Bahar F, Marriott JF. The effectiveness of computerised decision support on antibiotic use in hospitals: A systematic review. PLoS One. 2017;12:e0183062.

15. Baysari MT, Lehnbom EC, Li L, et al. The effectiveness of information technology to improve antimicrobial prescribing in hospitals: A systematic review and meta-analysis. Int J Med Inform. 2016;92:15-34.

16. Micallef $\mathrm{C}$, Chaudhry NT, Holmes AH, et al. Secondary use of data from hospital electronic prescribing and pharmacy systems to support the quality and safety of antimicrobial use: a systematic review. J Antimicrob Chemother. 2017;72:1880-5.

17. EU-JAMRAI. Work Package 7. List of revised guidelines; tools and implementation methods for antibiotic stewardship: Hospital care, https://eujamrai.eu/wp-content/uploads/2018/11/EUjamrai_D7.1_RevisedGuidelinesATBS_HOSPITALCare_WP7_2018.10.31_FV.pdf (2018, accessed 5 April 2019).

18. Public Health England. Start Smart-Then Focus. Antimicrobial Stewardship Toolkit for English Hospitals, https://www.gov.uk/government/publications/antimicrobial-stewardship-start-smart-then-focus (2015, accessed 13 November 2018).

19. Seaton RA, Nathwani D, Burton P, et al. Point prevalence survey of antibiotic use in Scottish hospitals utilising the Glasgow Antimicrobial Audit Tool (GAAT). Int J Antimicrob Agents. 2007;29:693-9.

20. European Centre for Disease Prevention and Control.

Point prevalence survey of healthcare-associated infections and antimicrobial use in European acute care hospitals

protocol version 5.3

European Centre for Disease Prevention and Control. Point prevalence survey of healthcare-associated infections and antimicrobial use in European acute care hospitals - protocol version 5.3. Stockholm. https://ecdc.europa.eu/en/publications-data/point-prevalence-surveyhealthcare-associated-infections-and-antimicrobial-use-3 (2016, accessed 15 May 2019).

21. UK Health and Social Care Information Centre. UK SNOMED CT

RF2

Full, Snapshot \& Delta

UK Health and Social Care Information Centre. UK SNOMED CT. Drug Extension. RF2: Full, Snapshot \& Delta, https://isd.digital.nhs.uk (2018).

22. World Health Organisation. The ICD-10 Classification of Mental and Behavioural Disorders. Clinical descriptions and diagnostic guidelines. Geneva, https://www.who.int/classifications/icd/en/bluebook.pdf (1993, accessed 26 November 2018).

23. Health and Social Care Information Centre. OPCS Classification of Interventions and Procedures Version 4.8 (SCCI0084 Amd 105/2015), https://digital.nhs.uk/data-and-information/information-standards/information-standards-and-data-collections-includingextractions/publications-and-notifications/standards-and-collections/scci0084-opcs-classification-of-interventions-and-procedures (2016, accessed 5 April 2019).

24. Nightingale PGG, Adu D, Richards NTT, et al. Implementation of rules based computerised bedside prescribing and administration: intervention study. BMJ. 2000;320:750-3.

25. UK Health and Social Care Information Centre. $d m+d$ Implementation Guide (Secondary Care) V5.0, https://www.nhsbsa.nhs.uk/sites/default/files/2017-02/Secondary_Care_Electronic_Prescribing_Implementation_Guidance_5_0.pdf (2015, accessed 15 April 2019).

26. European Committee on Antimicrobial Susceptibility Testing. EUCAST Expert Rules Version 3.1. Intrinsic Resistance and Exceptional Phenotypes Tables. 26 September. 2016, http://www.eucast.org/fileadmin/src/media/PDFs/EUCAST_files/Expert_Rules/Expert_rules_intrinsic_exceptional_V3.1.pdf (2016, accessed 1 May 2019).

27. Magiorakos A-P, Srinivasan A, Carey RB, et al. Multidrug-resistant, extensively drug-resistant and pandrug-resistant bacteria: an international expert proposal for interim standard definitions for acquired resistance. Clin Microbiol Infect. 2012;18:268-81. 
28. R Core Team. R: A Language and Environment for Statistical Computing, https://www.r-project.org/ (2018).

29. Wickham H. tidyverse: Easily Install and Load the 'Tidyverse', https://cran.r-project.org/package=tidyverse (2017).

30. dplyr: A Grammar of Data Manipulation

Wickham H, François R, Henry L, et al. dplyr: A Grammar of Data Manipulation, https://cran.r-project.org/package=dplyr (2019).

31. Chang W, Cheng J, Allaire J, et al. shiny: Web Application Framework for R. R package version 1.0.5., https://cran.r-project.org/package=shiny (2017).

32. Chang W, Ribeiro BB. shinydashboard: Create Dashboards with 'Shiny'. R package version 0.7.1., https://cran.r-

project.org/package=shinydashboard (2018).

33. Attali D, Almende BV, timevis. Create Interactive Timeline Visualizations in R. R package version 0.5.

34. Kunst J. highcharter: A Wrapper for the 'Highcharts' Library. R package version 0.7.0., https://cran.r-project.org/package=highcharter (2019).

35. Almende BV. vis.js: A dynamic, browser-based visualization library. JavaScript library version 4.21.0, http://visjs.org/ (2019).

36. Lim WS. Defining community acquired pneumonia severity on presentation to hospital: an international derivation and validation study. Thorax. 2003;58:377-82.

37. Ibrahim OM, Polk RE. Antimicrobial use metrics and benchmarking to improve stewardship outcomes: Methodology, opportunities, and challenges. Infect Dis Clin North Am. 2014;28:195-214.

38. Athanassa Z, Makris G, Dimopoulos G, et al. Early Switch to Oral Treatment in Patients with Moderate to Severe Community-Acquired Pneumonia. Drugs. 2008;68:2469-81.

39. Tabah A, Bassetti M, Kollef MH, et al. Antimicrobial de-escalation in critically ill patients: a position statement from a task force of the European Society of Intensive Care Medicine (ESICM) and European Society of Clinical Microbiology and Infectious Diseases (ESCMID) Critically III Patient. Intensive Care Med. Epub ahead of print 28 November 2019. DOI: 10.1007/s00134-019-05866-w.

40. Charani E, de Barra E, Rawson TM, et al. Antibiotic prescribing in general medical and surgical specialties: a prospective cohort study. Antimicrob Resist Infect Control. 2019;8:151.

41. Vlahović-Palčevski V, Gyssens IC. Quality Indicators and Quantity Metrics of Antibiotic Use. In: Antimicrobial Stewardship. Elsevier, pp. $29-37$.

42. Beaudoin M, Kabanza F, Nault V, et al. An Antimicrobial Prescription Surveillance System that Learns from Experience. Al Mag. $2014 ; 35: 15$.

43. Lovis C, Colaert D, Stroetmann VNN. DebugIT for patient safety - improving the treatment with antibiotics through multimedia data mining of heterogeneous clinical data. Stud Health Technol Inform. 2008;136:641-6.

44. Morales A, Cánovas-Segura B, Campos M, et al Proposal of a Big Data Platform for Intelligent Antibiotic Surveillance in a Hospital. In: Luaces O, Gámez JA, Barrenechea E, et al, editors Advances in Artificial Intelligence. 17th Conference of the Spanish Association for Artificial Intelligence, CAEPIA 2016, Salamanca, Spain, September 14-16, 2016, pp. 261-270.

45. UK Department of Health and Social Care. Tackling antimicrobial resistance 2019-2024. The UK's five-year national action plan, https://www.gov.uk/government/publications/uk-5-year-action-plan-for-antimicrobial-resistance-2019-to-2024 (2019, accessed 8 April 2019).

46. NHS Digital. Clinical Information Standards (beta). 21 January 2019, https://digital.nhs.uk/about-nhs-digital/our-work/nhs-digital-data-andtechnology-standards/clinical-information-standards (2019, accessed 8 November 2019).

47. 10.1002/14651858.CD000259.pub3

Ivers N, Jamtvedt G, Flottorp S, et al. Audit and feedback: effects on professional practice and healthcare outcomes. Cochrane Database Syst Rev. Epub ahead of print 13 June 2012. DOI: 10.1002/14651858.CD000259.pub3.

48. Brehaut JC, Colquhoun HL, Eva KW, et al. Practice Feedback Interventions: 15 Suggestions for Optimizing Effectiveness. Ann Intern Med. 2016;164:435.

49. Van Santen KL, Edwards JR, Webb AK, et al. The standardized antimicrobial administration ratio: A new metric for measuring and comparing antibiotic use. Clin Infect Dis. 2018;67:179-85.

50. Yardley L, Morrison L, Bradbury K, et al. The Person-Based Approach to Intervention Development: Application to Digital Health-Related Behavior Change Interventions. J Med Internet Res. 2015;17:e30.

51. Berends MS, Luz CF, Friedrich AW, et al. AMR - An R Package for Working with Antimicrobial Resistance Data. bioRxiv $2019 ; 810622$.

\section{Figures}




\section{Antimicrobial therapy overview}

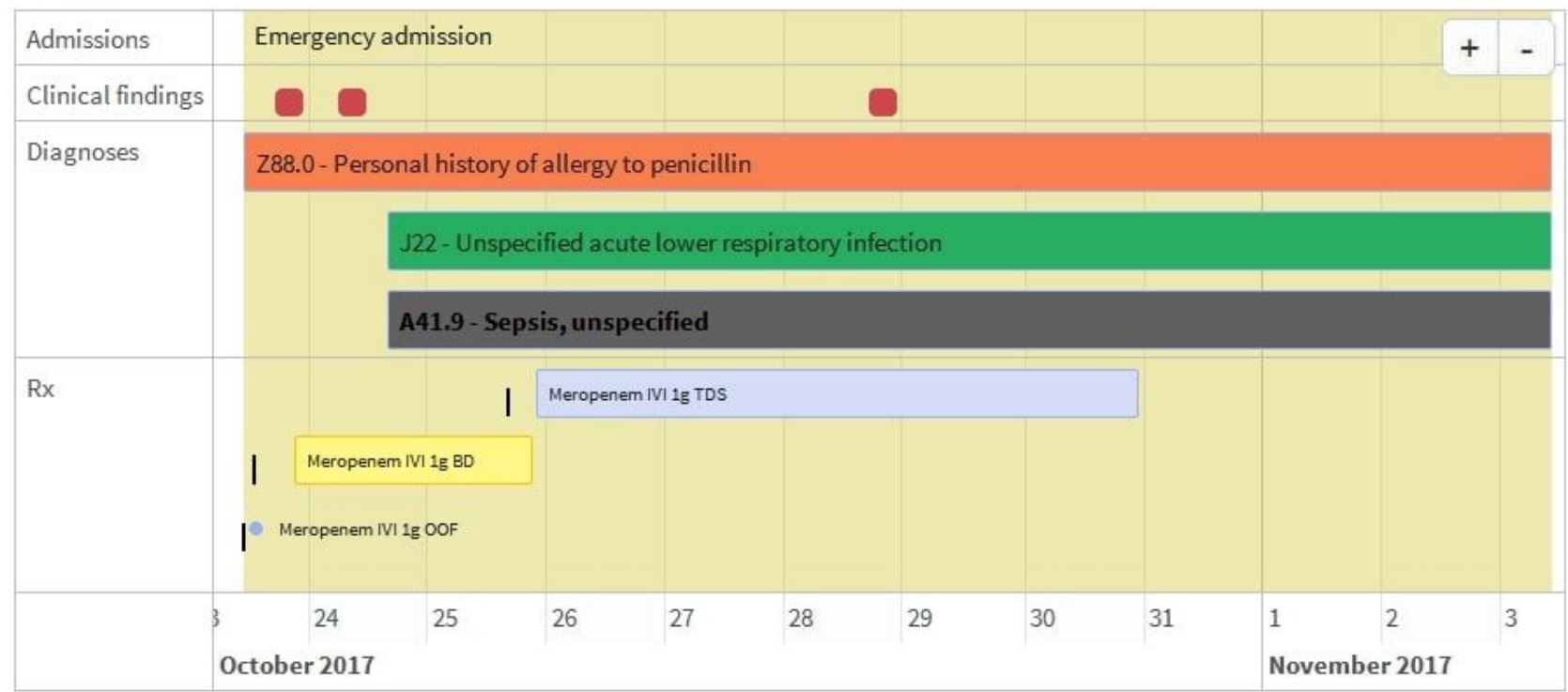

Admissions Diagnoses Procedures Prescriptions Investigations

- Chous 10 - nntrine

$$
\text { Cnsols. }
$$

\section{Figure 1}

Screenshot of antimicrobial timeline visualisation

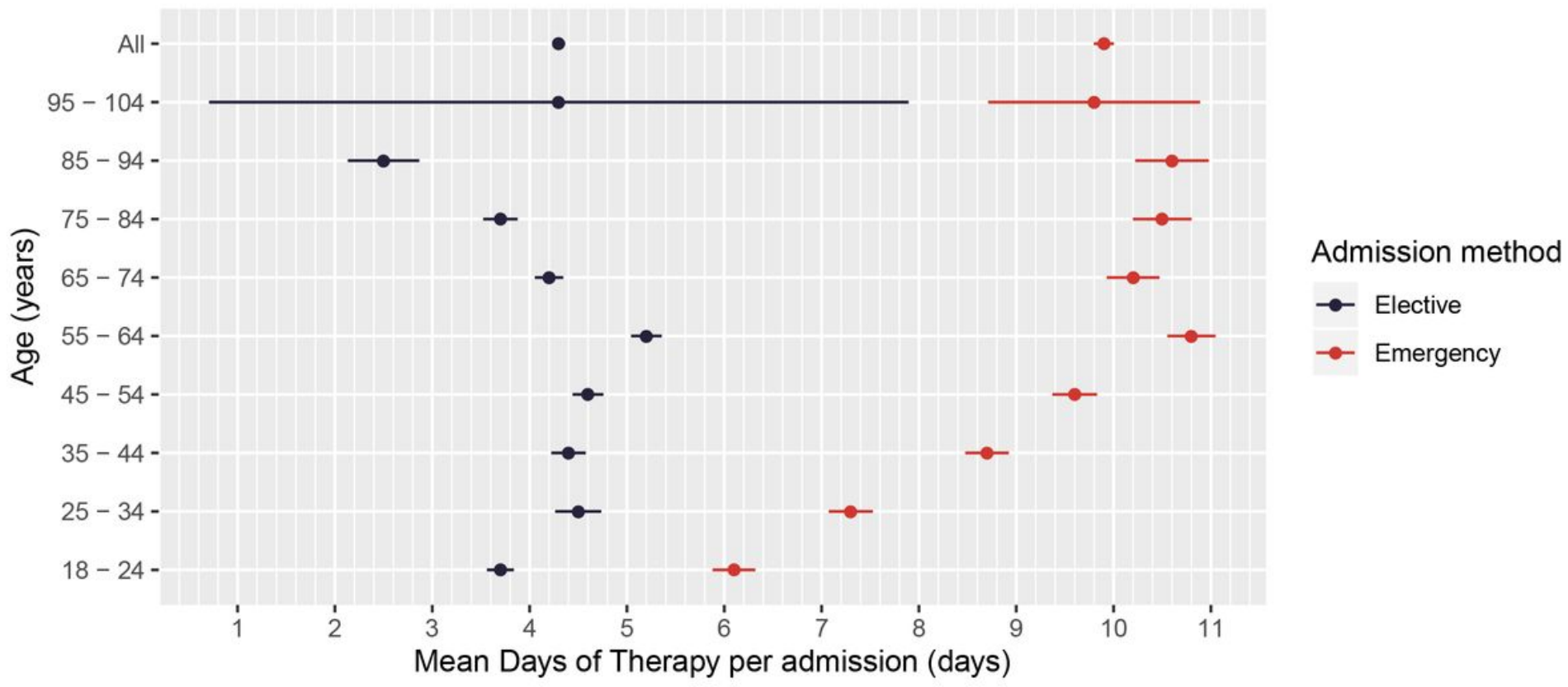

\section{Figure 2}

Mean and $95 \% \mathrm{Cl}$ of the total DOT per admission in patients receiving antimicrobials at any point during an admission (September 2017-August 2018) 


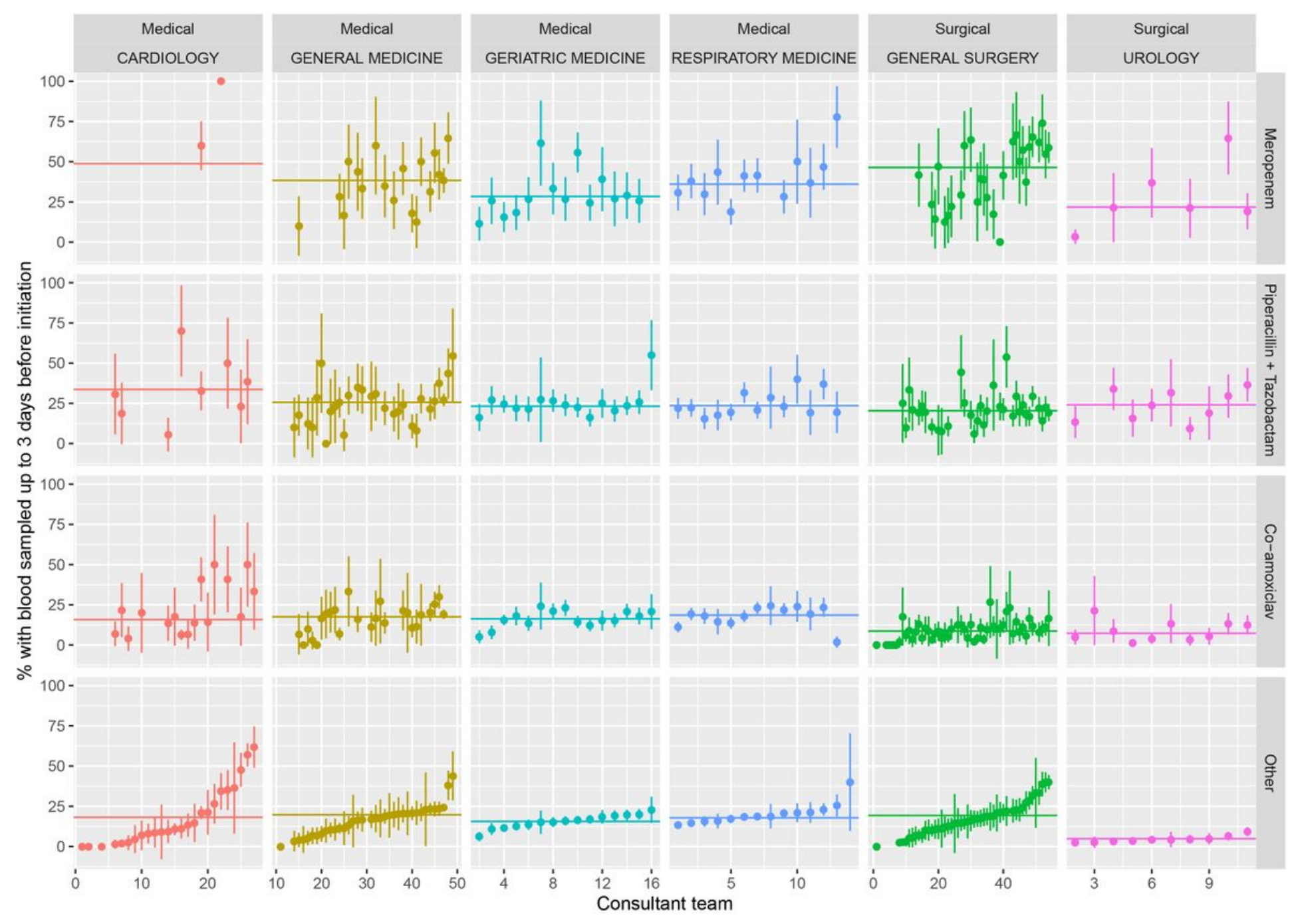

\section{Figure 3}

Point and 95\% confidence interval estimates of the proportion of prescriptions initiated with a blood culture sampled in the three days leading up to initiation of prescription and/or therapy by consultant team by specialty by drug type in six selected specialities (September 2017-August 2018)

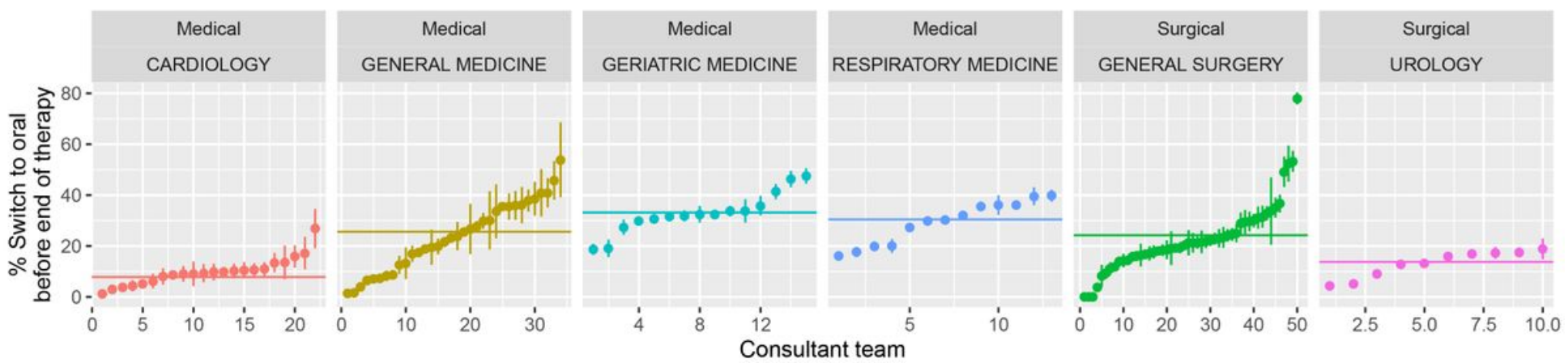

\section{Figure 4}

Point and $95 \%$ confidence interval estimates of the proportion of intravenous therapy converted to oral therapy ranked by consultant team by specialty (September 2017-August 2018) 

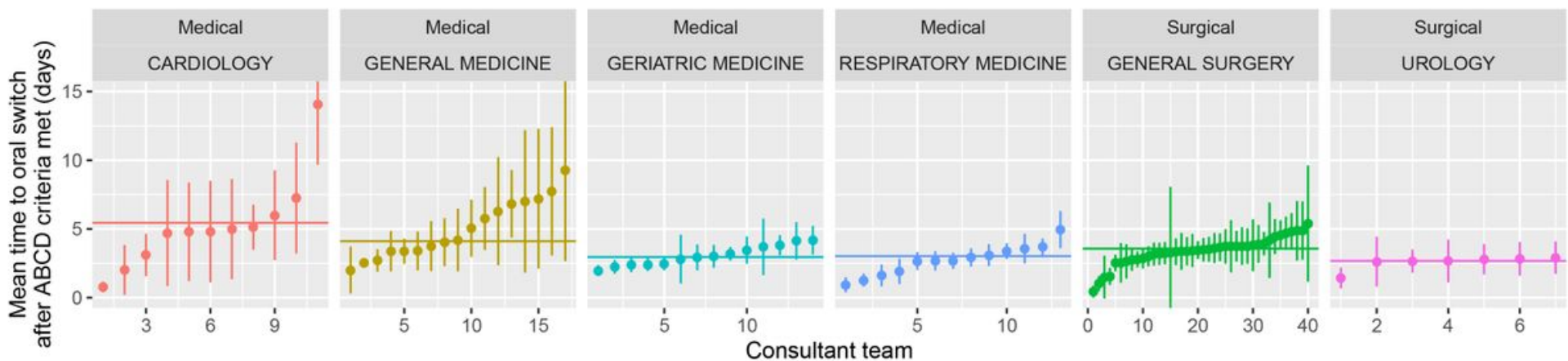

\section{Figure 5}

Point and 95\% confidence interval estimates of the mean time (days) elapsed between ABCD criteria being met and conversion to oral therapy, ranked by consultant team by specialty (September 2017-August 2018)

\section{Supplementary Files}

This is a list of supplementary files associated with this preprint. Click to download.

- supplement9.docx

- supplement10.xlsx

- supplement11.docx

- supplement12.docx

- supplement13.docx

- supplement14.docx 